\title{
Paysages bioculturels et biomimétisme à un niveau écosystémique
}

Do farmers use, and imitate, self-organizing resource-concentration mechanisms driven by non-human engineers in constraining environments? Biocultural landscapes and biomimicry at the ecosystem level

Doyle McKey

\section{CpenEdition}

\section{Journals}

Electronic version

URL: http://journals.openedition.org/actesbranly/667

ISSN: $2105-2735$

Publisher

Musée du quai Branly Jacques Chirac

Electronic reference

Doyle McKey, «Paysages bioculturels et biomimétisme à un niveau écosystémique », Les actes de colloques du musée du quai Branly Jacques Chirac [Online], 6 | 2016, Online since 20 January 2016, connection on 08 September 2020. URL : http://journals.openedition.org/actesbranly/667

This text was automatically generated on 8 September 2020

(c) Tous droits réservés 


\section{Paysages bioculturels et biomimétisme à un niveau écosystémique}

Do farmers use, and imitate, self-organizing resource-concentration mechanisms driven by non-human engineers in constraining environments? Biocultural landscapes and biomimicry at the ecosystem level

\section{Doyle McKey}

1 Je vais vous parler de deux exemples de milieux très contraignants, où les organismes ont façonné des réponses à ces contraintes, et où, de façon intéressante, les êtres humains ont façonné des réponses assez similaires. Cette similarité nous interpelle.

Dans le premier exemple, il s'agit des milieux semi-arides. Partout dans le monde, dans une diversité de milieux, il y a des structures d'origine naturelle qui sont spatialement régulières, construites pour la plupart par des organismes. La figure 1 montre les types de paysages réguliers rencontrés dans les milieux semi-arides. Par exemple, la brousse tachetée dans le Sahel, dont certaines zones en Afrique semi-aride et ailleurs dans le monde. Il peut y avoir des régimes un peu différents, des patrons de labyrinthe et, sur les pentes, de la brousse tigrée, avec des bandes de végétation suivant les lignes de contour. Les patterns réguliers à grande échelle dans les paysages résultent des processus à l'échelle locale. Ce phénomène s'appelle l'auto-organisation spatiale. 
Figure 1. Paysages spatialement auto-organisés dans des milieux semi-arides.

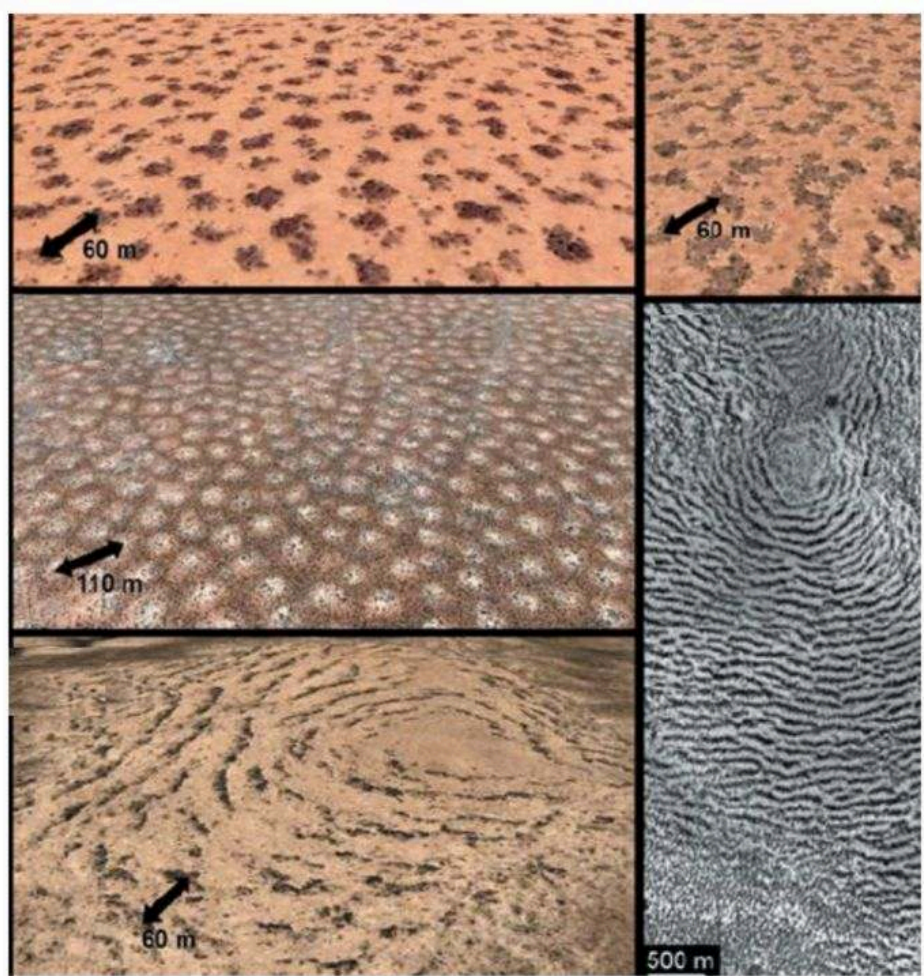

À gauche, en haut et au milieu, apparaissent deux exemples de «brousse tachetée » qu'on trouve dans des paysages plans. En bas à gauche, et sur la droite, deux exemples de «brousse tigrée » typique des paysages en pente. En haut à droite, enfin, nous pouvons voir des taches de végétation en labyrinthe.

Adapté d'une figure de Deblauwe et al. (2008)

3 Ces processus auto-organisants sont mis en marche par les organismes qu'on qualifie d'ingénieurs d'écosystème. Dans ces exemples, les organismes moteurs de cette autoorganisation sont des plantes. Ce pattern régulier n'est pas le projet d'une communauté de plantes, mais une propriété émergente de l'interaction de chaque plante individuelle avec son milieu. Avant de voir comment cela fonctionne, je voudrais vous montrer que dans des milieux similaires, dans la même région, il y a aussi des paysages spatialement très réguliers, qui sont similaires mais qui sont d'origine culturelle, qui sont planifiés et construits par les agriculteurs. C'est l'exemple du système zaï (figure 2), qui, en Afrique de l'Ouest, « imite » d'une certaine façon la brousse tachetée. 
Figure 2. Un exemple du système zaï, qui ressemble, dans sa forme et dans son fonctionnement, à la brousse tachetée.

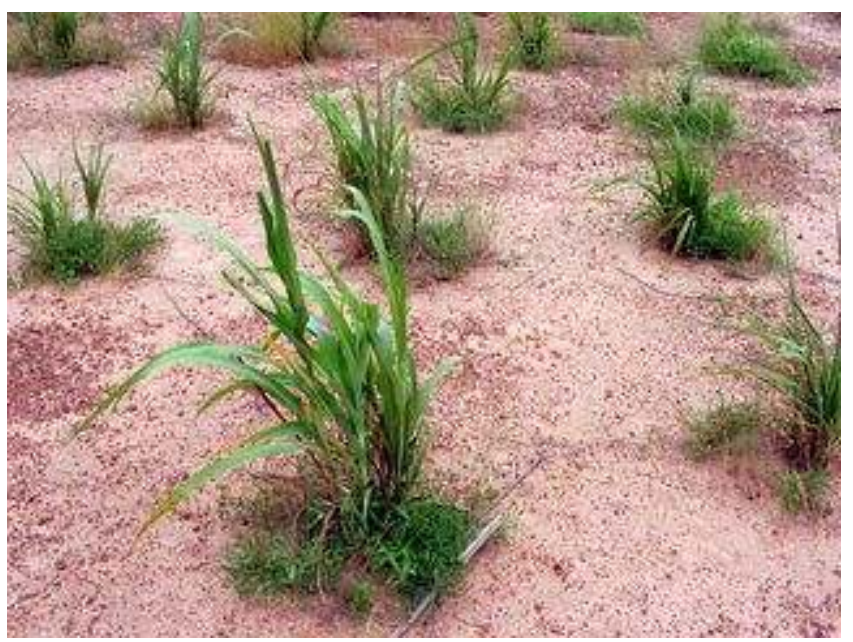

Illustration prise du site http://en.howtopedia.org/wiki/How_to_Start_Culture_in_Zai_Holes

paysages, d'origine culturelle et naturelle, non seulement ont une apparence similaire, mais ils fonctionnent aussi de façon très similaire comme écosystèmes. On peut les qualifier de diverses manières. Ce sont des objets complexes. Mais les paysages d'origine naturelle (le système zaï, figure 2) possèdent au moins une caractéristique sur laquelle on peut s'accorder : ce sont des artefacts, parce qu'ils sont produits par des agriculteurs.

5 Je souhaite poser la question des relations entre ces artefacts et les paysages similaires d'origine naturelle, les structures biogéniques créées par d'autres êtres vivants dans des milieux similaires.

6 Comment fonctionne l'auto-organisation spatiale dans les écosystèmes ?

7 Il y a une diversité de tels paysages réguliers, auto-organisés, dans les régions semiarides, comme ailleurs. Le principe est le suivant. Dans ces milieux, il y a une ressource ou un facteur environnemental critique qui contraint la répartition des organismes. Certains organismes, qu'on qualifie d'ingénieurs, ont des effets physiques ou chimiques sur la structure de l'habitat, ils modifient la répartition de cette ressource et la concentrent dans des tâches, avec des espaces dépourvus de ressources entre ces tâches. Dans les milieux semi-arides, la ressource critique est l'eau.

8 Alan Turing, vers la fin de sa vie, a cherché à expliquer la formation de patrons réguliers dans le pelage des animaux. Il a développé un modèle pour la morphogénèse du pelage, qui implique des rétroactions dont le sens (positif ou négatif) dépend de l'échelle. Il a imaginé une situation où on a une rétroaction positive sur la production de pigments dans le pelage à courte échelle et une rétroaction négative sur la production de pigments à une échelle légèrement plus grande. Si on est aussi bon en mathématiques que Turing, on peut générer des patrons réguliers avec cette combinaison assez simple. Au final, le même mécanisme explique la formation de motifs réguliers dans le pelage des animaux et dans les écosystèmes de type brousse tachetée et brousse tigrée.

9 Comment ces mécanismes produisent-ils des patrons dans ces écosystèmes semiarides? Ce sont encore des rétroactions dont le sens positif ou négatif dépend de l'échelle. Quelles sont ces rétroactions? Dans ces milieux semi-arides, les plantes, par 
leurs racines, créent des sols poreux. Mais entre les plantes, le sol est encroûté. Aussi, entre les plantes, les racines s'étendent sur un rayon beaucoup plus grand que la canopée des plantes. Près d'une plante, le sol est poreux et l'eau s'infiltre. Ce sol humide favorise l'établissement d'autres plantes. C'est la rétroaction positive à courte échelle. Plus loin de la plante, l'eau coule de façon horizontale sur le sol encroûté jusqu'à arriver à proximité d'une plante, où il s'infiltre dans le sol poreux. L'eau est ainsi assimilée par les racines, et le sol entre les plantes reste sec, empêchant l'établissement de plantes. En quelque sorte, un groupe de plantes peut s'établir uniquement à partir d'une distance minimum d'autres plantes. Cela conduit à un espacement régulier avec une distance entre les taches déterminée par l'échelle des processus mis en marche par les ingénieurs d'écosystème. Le sol encroûté entre les plantes récolte l'eau qui fuit latéralement sur les plantes, et une fois que l'eau arrive latéralement jusqu'à une plante, elle s'infiltre immédiatement. L'eau est stockée là où il y a déjà des plantes. Le sol encroûté, en combinaison avec les racines des plantes, crée un système qui concentre l'eau là où les plantes se trouvent déjà.

10 Les patrons fournis dépendent de la topographie. Sur les paysages plans, l'eau coule horizontalement. On parle d'isotropie, car il n'y a pas d'orientation favorisée : l'eau coule dans tous les sens. Cela donne lieu aux brousses tachetées. Sur des pentes, l'eau coule le long des pentes, on parle d'anisotropie, car il y a une orientation favorisée. Cela produit des bandes de végétation qui suivent les contours : la brousse tigrée. L'espace entre les taches est déterminé par la surface nécessaire pour récolter suffisamment d'eau pour laisser pousser une autre bande de plantes.

$11 \mathrm{Au}$ niveau de l'écosystème, le résultat de tous ces processus est que les plantes, en interaction avec le milieu physique, concentrent l'eau dans les taches, et enlèvent l'eau entre les taches. Ce mécanisme de concentration de ressources permet aux plantes de pousser dans des endroits où, si l'eau de pluie était répartie régulièrement, les plantes ne pourraient pas pousser. C'est uniquement par ce mécanisme de concentration de ressources que les plantes persistent. En fait, le système zaï, qui a été inventé par des agriculteurs dans le Sahel, intègre ces mêmes mécanismes de concentration de ressources qui produisent les paysages réguliers d'origine naturelle dans les mêmes milieux.

12 Le système zaï était une réponse à un problème: comment réhabiliter les sites dégradés, dévégétalisés, dénués de plantes? Une recommandation fréquente des agronomes était de détruire ce sol encroûté et de laisser pénétrer l'eau. Les paysans ont refusé cette solution. Pour eux, il fallait laisser le sol encroûté entre des plantes et faire un sol poreux uniquement là où on va mettre des plantes. Leur génie était de préserver ce sol encroûté entre les plantes et l'utiliser pour récolter la pluie qui va couler vers les plantes, où elle va s'infiltrer. De façon frappante, les agriculteurs forment des zaï tachetés sur les zones planes, et des zaï tigrés sur les pentes, exactement comme le fait la nature. Ce sont vraiment des similarités frappantes.

Les agronomes ont décrit les mécanismes de rétroaction qui sous-tendent le fonctionnement de ce système. Mais ils sont une communauté de recherche complètement différente des écologues théoriciens qui travaillent sur les mécanismes d'auto-organisation. Rien ne suggère qu'ils se parlent les uns aux autres. Personne n'a jamais demandé aux agriculteurs comment ils réfléchissent à ces paysages. Si agronomes, agriculteurs et écologues se parlaient tous entre eux, on trouverait certainement qu'on aurait beaucoup de choses à se dire. 


\section{Les paysages à buttes de l'Amérique du Sud}

14 analogies frappantes.

Dans ce milieu, le problème n'est pas le manque d'eau, mais l'excédent d'eau durant une partie de l'année. On arrive pourtant à des conséquences similaires au niveau de l'organisation du paysage. En Amérique du Sud, dans le Mato Grosso, dans le Pantanal, dans les llanos del Beni en Bolivie, dans les llanos de l'Orénoque en Colombie et au Venezuela, il y a une diversité de paysages très réguliers, dans ces savanes saisonnièrement inondées. On parle de paysages à buttes. Tous ces paysages sont d'origine naturelle; leurs constructeurs sont des ingénieurs d'écosystèmes comme des arbres, des termites, des vers de terre, etc. La figure 3 montre un exemple.

Figure 3. La photo, prise dans les Llanos de l'Orénoque de Colombie, montre un exemple des " paysages à buttes » d'origine naturelle qui se trouvent dans des savanes inondables d'Amérique du Sud.

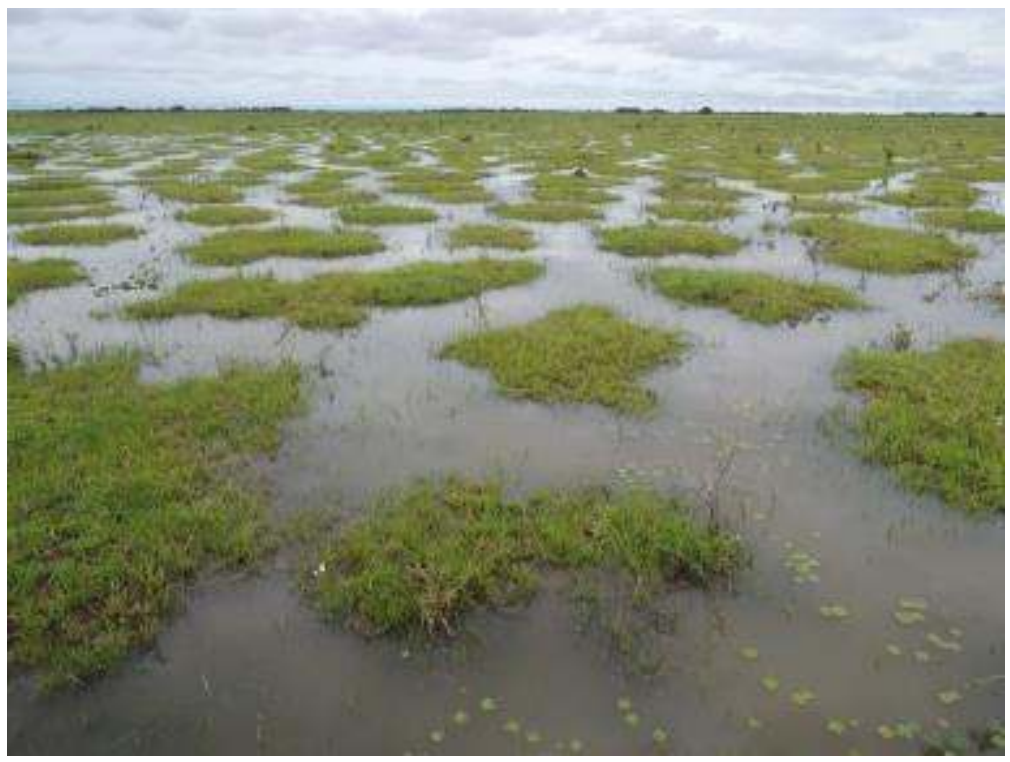

Photographie : Doyle McKey 
Figure 4. Vestiges de champs surélevés précolombiens, Guyane.
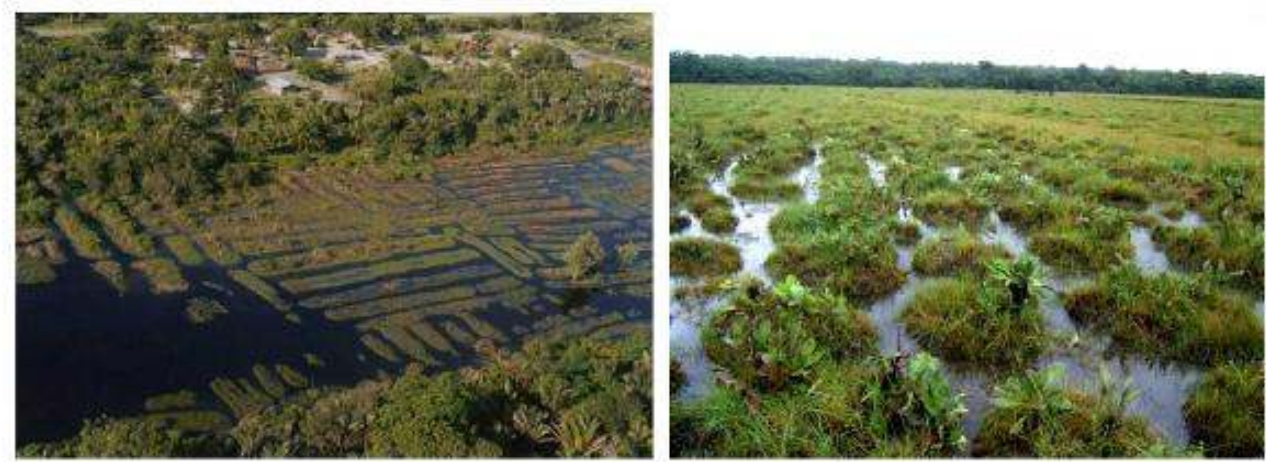

À gauche, des champs surélevés en forme de billons, arrangés en damier. Photographie : Stéphen Rostain. À droite, des champs surélevés en forme de monticules ronds. Photographie : Doyle McKey

Dans certains de ces milieux, on voit d'autres paysages à buttes, mais qui sont d'origine anthropique. En Amérique du Sud, ce sont tous des vestiges de l'agriculture précolombienne sur champs surélevés. Les systèmes connus sont repartis dans des savanes saisonnièrement inondées sur le pourtour de l'Amazonie. Nous les avons étudiés dans les savanes côtières de la Guyane française. Les paysages à buttes illustrés dans la figure 4 sont d'origine humaine. Nous avons vérifié cela avec des données géoarchéologiques, archéobotaniques et archéologiques. Pour certains paysages, leur origine anthropique est très claire, indiquée par des billons linéaires, souvent organisés en damier (figure 4, photo de gauche). Cependant, d'autres champs surélevés sont des monticules ronds, et leur ressemblance aux paysages d'origine naturelle, comme ceux montrés dans la figure 3, est plus frappante (figure 4, photo de droite). On connaît peu de choses sur le fonctionnement de ces paysages à buttes - d'origine naturelle ou d'origine anthropique - dans les savanes saisonnièrement inondées. J'ai trouvé peu d'autres chercheurs qui connaissent ces zones et qui s'y intéressent. On peut donc se demander : de quelle manière ont-elles été formées? 
Figure 5. Capture d'écran de Google Earth ${ }^{\mathrm{TM}}$ illustrant comment nous avons commencé à trouver nos sites d'étude dans les surales.

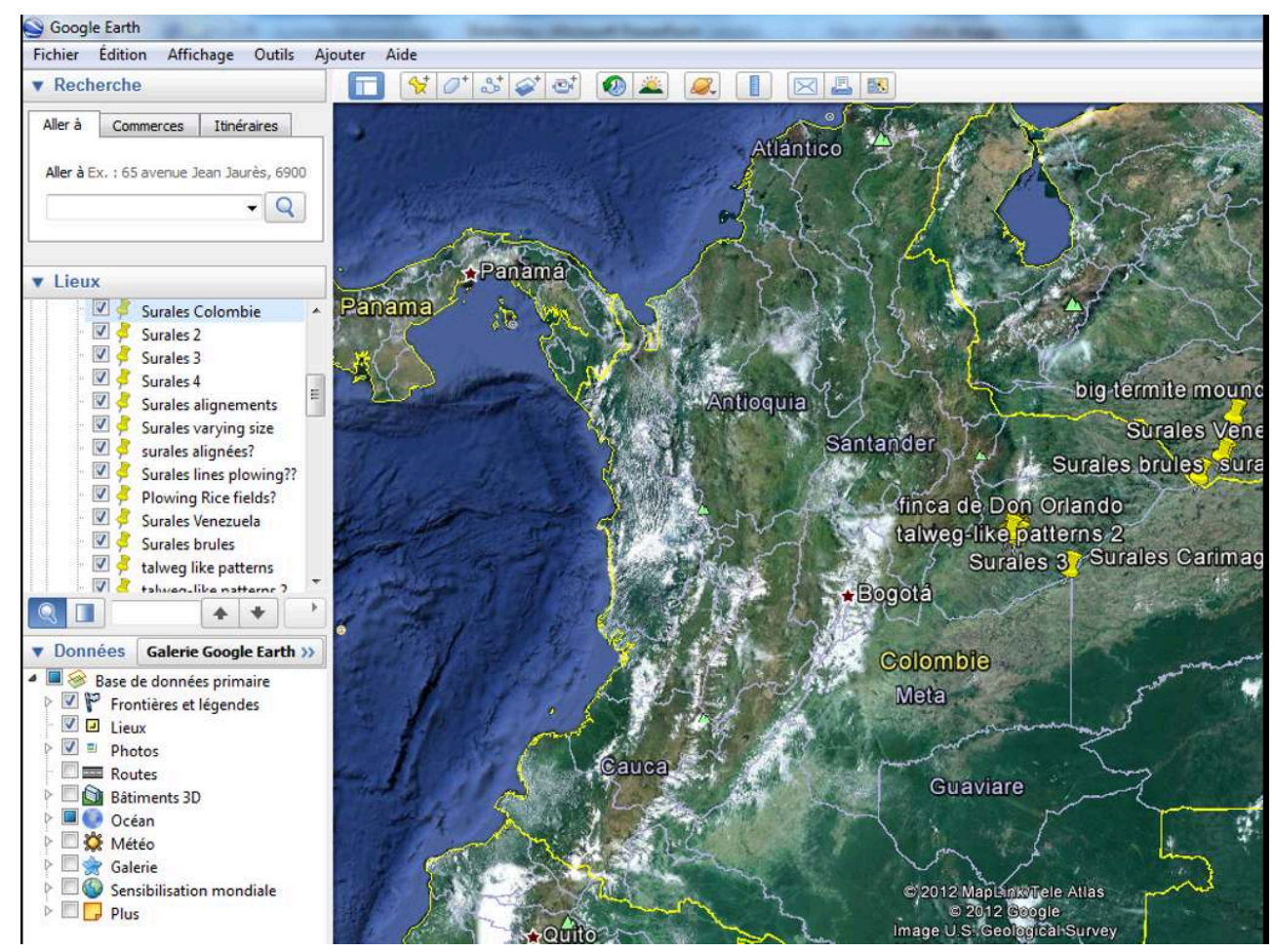

Quelques articles publiés fournissaient des points géoréférencés pour ces paysages. Nous avons cherché des images sur Google Earth ${ }^{\mathrm{TM}}$ montrant ces structures, pour finalement trouver des exemples prometteurs accessibles par la route.

Nous avons quitté la Guyane française et nous sommes allés dans les llanos de l'Orénoque pour étudier un paysage spectaculaire, d'origine entièrement naturelle, les surales, qui sont des paysages à buttes s'étendant sur des kilomètres carrés et constitués d'excréments de vers de terre. D'abord, il fallait les trouver. Certains écologues ont parlé de ces buttes. On a trouvé des points sur Google Earth ${ }^{\mathrm{TM}}$ (figure 5). Très rapidement, un point s'est révélé être suffisamment près d'une route. Ce point, c'était la finca de don Jorge Niño (figure 6). 
Figure 6. Lors de notre première mission sur le terrain, l'un de ces sites prometteurs s'est révélé être la finca de don Jorge Niño

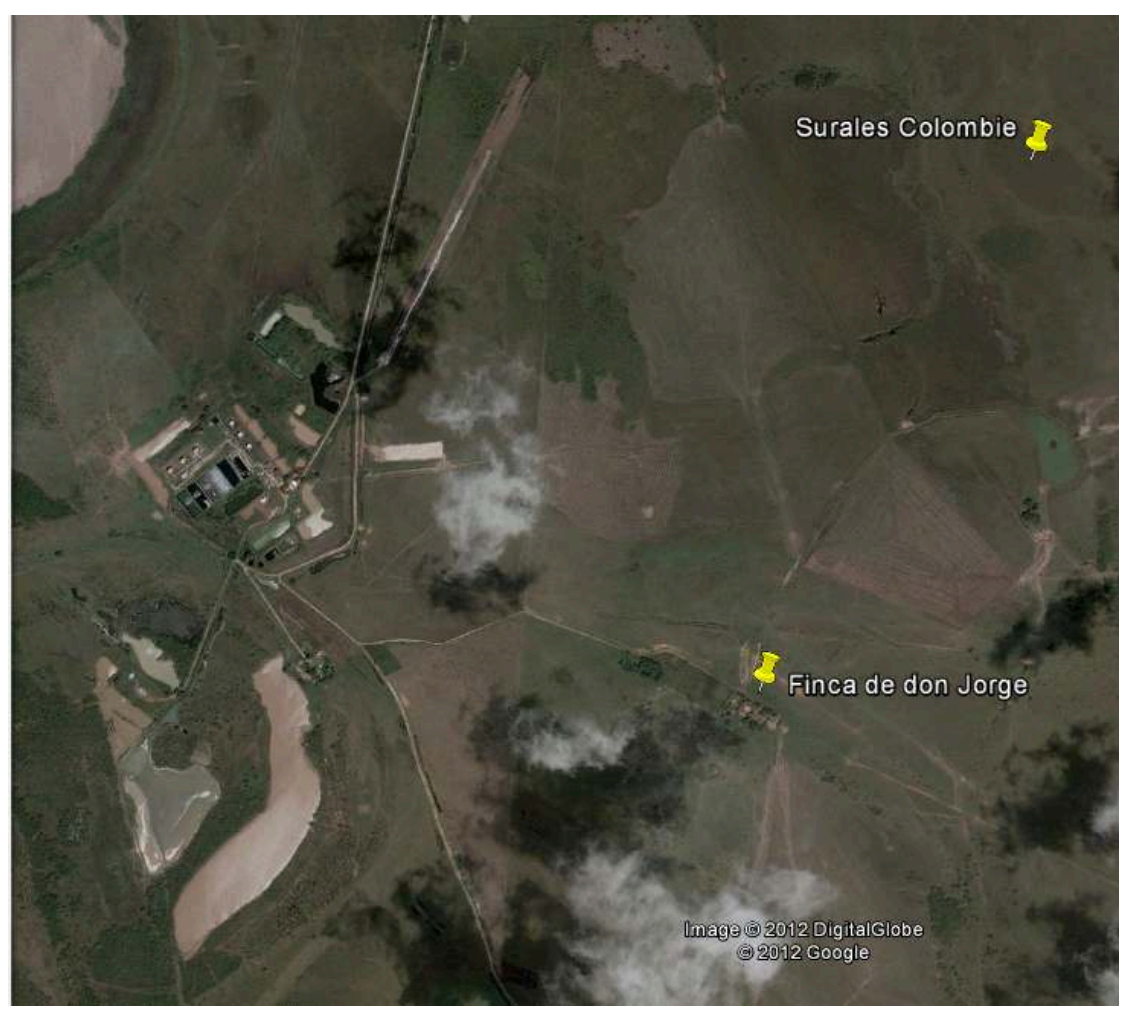

Que sont les surales? Ce sont des paysages à buttes construits par les vers de terre. Elles commencent « bébés ». Il y a une longue séquence de développement. Ce sont de petites tours créées par les vers de terre dans les endroits faiblement inondés (figure 7). Les vers de terre, au début, ne peuvent pas faire des buttes hautes; ils font donc des petits tours. Et c'est uniquement dans les parties faiblement inondées qu'ils peuvent 'prendre pied'. La tour devient peu à peu une butte, qui se développe avec le temps. Ce ver de terre est assez spécifique. Pour l'instant, nous n'en avons trouvé que des juvéniles et nous ne connaissons pas leur taille adulte. Ces grands vers déplacent beaucoup de terre et c'est la raison pour laquelle ils ont créé des vastes étendues de ces paysages.

Figure 7. Les buttes dans les paysages de surales commencent à partir de tours faites par des vers individuels.

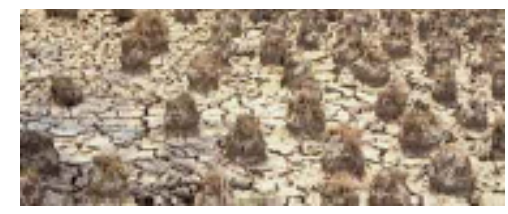

Photographie : Doyle McKey

Comment poussent les surales? Les vers de terre mangent le sol riche en matière organique des macrophytes aquatiques dans ces plaines inondées. Ils transportent le sol dans des tours parce qu'il faut qu'ils arrivent à la surface pour respirer. Comme ils reviennent toujours au même endroit, les tours forment des buttes.

20 Les petites buttes, comme celles illustrées dans la figure 3 (et dans la vue aérienne, figure 8 , photo de gauche), s'agrègent pour former des buttes plus grandes, comme 
celle montrée dans la figure 8 , photo de droite, qui est composée d'au moins huit petites buttes. Les surales deviennent plus grands, plus hauts, couverts de graminées, mais aussi d'arbustes. Plus hautes sont les buttes, plus grande est la quantité du sol enlevée du bassin autour, donc plus profond est le bassin.

Figure 8. Dans les paysages surales, les petites buttes, comme celles montrées dans la Figure 3 et dans la vue aérienne ici (photo de gauche, photographie : Delphine Renard), s'agrègent pour former des buttes plus grandes (photo de droite, photographie : Doyle McKey)
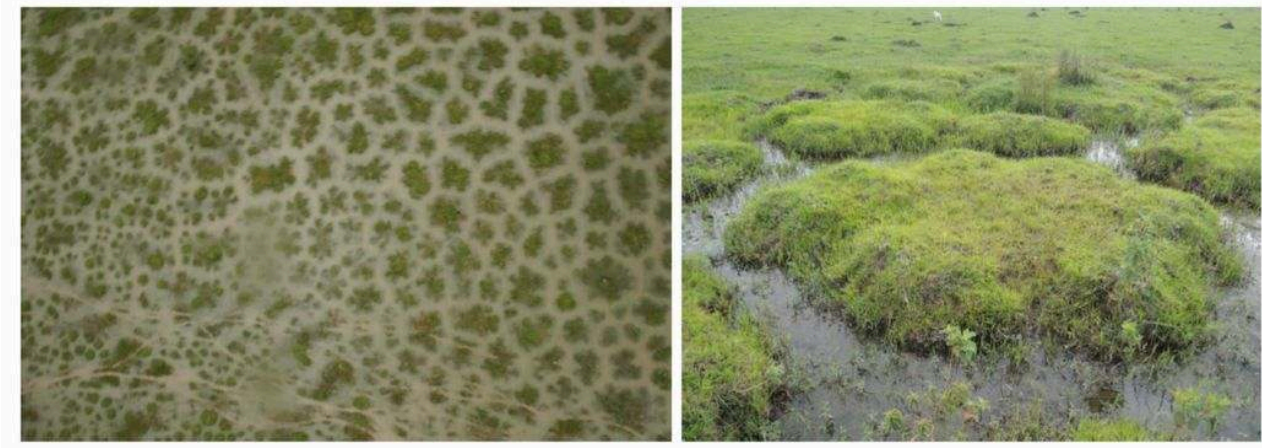

Y a-t-il des parallèles avec d'autres paysages réguliers, comme ceux des paysages semiarides? Quels mécanismes conduisent à cette régularité spatiale chez les surales? On ne sait pas. Notre hypothèse de travail est que la ressource critique ici, ce n'est pas l'eau, mais plutôt le sol oxygéné. Les ingénieurs dans ce cas ne sont pas des plantes, mais des vers de terre qui modifient la répartition du sol oxygéné, créant des taches riches en cette ressource, les buttes ou monticules, et des espaces dépourvus de cette ressource, le bassin inondé entre les buttes. Cela marche à peu près comme cela, dans un milieu faiblement inondé, avec une hauteur d'eau de quelques décimètres, le ver mange le sol et fait sa petite tour au-dessus du niveau de l'eau pour respirer. Mais comme il fourrage près de la butte, il bouge le sol du bassin vers la butte. Plus la butte devient haute, plus le bassin devient profond. Par conséquent, moins il y a possibilité qu'un autre ver puisse s'établir dans cet espace. Le sol constamment porté à la butte est la rétroaction positive (des vers sur la disponibilité de sol bien aéré) à courte échelle. La profondeur grandissante du bassin est la rétroaction négative sur la topographie, à échelle spatiale un peu plus grande. On pense que c'est ce mécanisme - tout à fait analogue à celui postulé par Turing - qui crée un espacement minimum entre les buttes, et donc la régularité.

Dans ces mêmes milieux, on trouve parfois à côté de ces paysages d'origine naturelle des vestiges de paysages à champs surélevés faits par les humains. Quand ces paysages d'origine naturelle et humaine coexistent, est-ce qu'il y a des interactions entre les deux? Je n'ai pas le temps de vous parler des llanos del Beni (connus aussi comme les llanos de Moxos). C'est là où il y a la plus grande diversité de paysages à buttes d'origine naturelle et culturelle, et nos observations indiquent des interactions fascinantes. Par exemple, les structures naturelles peuvent être intégrées dans le paysage anthropisé. Tirant profit du fait que les termites aient déjà construit des structures surélevées et concentré des ressources dedans, les agriculteurs peuvent aménager un groupe de termitières pour faire une plateforme de plantation.

Dans d'autres régions, il manque des ingénieurs du sol spécialisés, comme le vers de terre géant des surales, ou les termites constructeurs de buttes dans les llanos del Beni. Néanmoins, ces ingénieurs peu spécialisés, même s'ils ne construisent pas des buttes 
eux-mêmes, peuvent toujours agir sur les buttes construites par des ingénieurs humains. Par exemple, dans les savanes côtières de Guyane, où existent des vestiges de champs surélevés, les interactions entre ces anciens artefacts humains abandonnés depuis au moins 500 ans, et les êtres vivants, ces ingénieurs peu spécialisés, façonnent l'écologie de ces paysages aujourd'hui. Comme écologues, nous sommes arrivés à l'étude de ce système avec une question simple. Il y a trois ou quatre mètres de pluies torrentielles tous les ans. Les paysages sont fréquemment brûlés pendant la saison sèche, enlevant une partie de la couverture végétale. Comment ces buttes arrivent-elles à persister contre l'érosion, malgré leur abandon depuis des siècles?

Quand on se promène dans ce paysage, on voit que toutes ces buttes d'origine anthropique sont colonisées par des insectes sociaux, des vers de terre et des plantes. Ces organismes et leurs structures biogéniques - les racines et les agrégats autour des racines, leur porosité, les tas de détritus des insectes sociaux, les turricules de vers de terre - sont tous concentrés sur les buttes abandonnées. En fait, les ingénieurs des écosystèmes colonisent d'un point de vue préférentiel ces buttes qui, durant la saison des pluies, leur fournissent un sol bien drainé, une ressource clé dans ces milieux souvent inondés.

Les insectes sociaux établissent toujours leurs nids sur ces parties élevées, les buttes. Ce sont des "central place foragers»: ils amènent plein de choses sur les buttes. Par exemple, la fourmi champignonniste Acromyrmex octospinosus (figure 9) amène des kilos de végétaux pour nourrir les champignons qu'elle cultive. Une fois que le champignon a "digéré » les tissus des végétaux amenés par les fourmis, ces derniers déposent les détritus en dehors du nid, toujours sur la butte. Notre hypothèse, soutenue par un certain nombre de données, est que ces ingénieurs déposent de la matière sur les buttes abandonnées et modifient les propriétés des sols, les rendant plus stables et plus poreux. Cette déposition de matière sur les buttes, et la stabilisation des buttes contre l'érosion par les ingénieurs naturels, expliquent pourquoi ces buttes, abandonnées depuis cinq cent ans, sont encore là.

Figure 9. Les ouvrières de la fourmi champignonniste Acromyrmex octospinosus amènent des fragments de végétaux pour nourrir leur champignon symbiotique dans leur nid, situé sur une butte (un ancien champ surélevé ; photo de gauche, photographie : Delphine Renard). Le détritus est ensuite déposé hors du nid, sur un tas qui lui aussi est situé sur la butte (photo de droite, photographie : Doyle McKey)
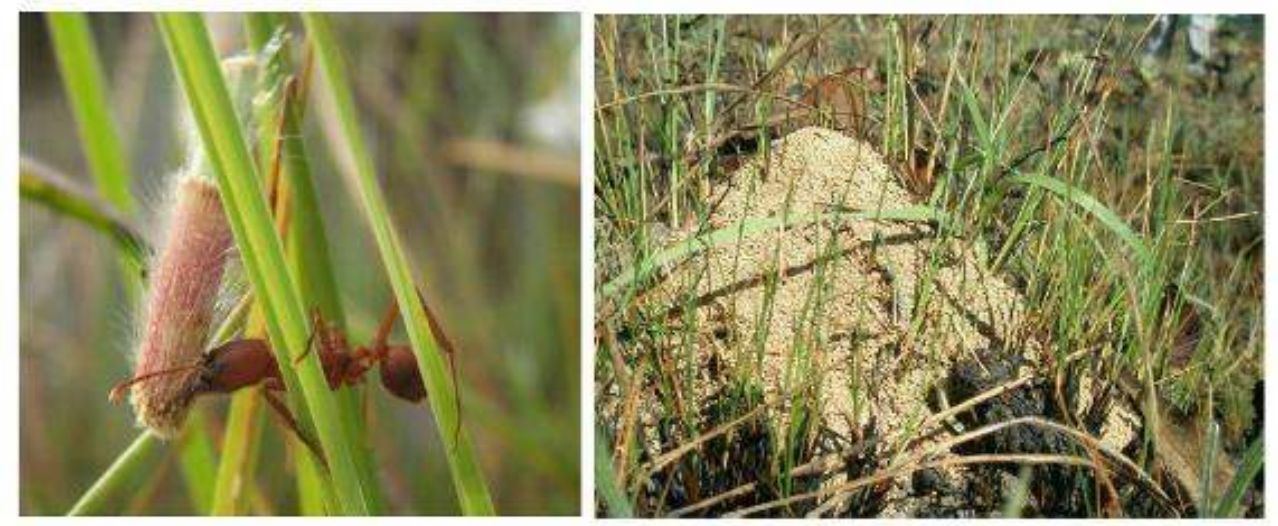

Est-ce que des interactions comme celles-là ont joué un rôle quand le système était actif? Vraisemblablement oui, parce que dans ces systèmes, comme d'autres en Afrique, il était probablement nécessaire d'avoir des jachères pour laisser le sol au 
repos. Ces jachères étaient indispensables pour reconstituer la matière organique et la structure du sol et aussi pour contrôler les ennemis des cultures. Durant la jachère, les populations des pestes et des pathogènes qui auraient accumulés localement sur les cultures vont diminuer, par manque de nourriture. On peut poser la question: construire ces buttes demande beaucoup de travail. Durant la jachère, est-ce que tout se décompose? En fait, ces champs en Guyane ont été en jachère depuis 500 ans et ils sont toujours là. Un Occidental pourrait être tenté de dire que les agriculteurs ont délocalisé le travail à une force d'ouvriers très bon marché : les vers de terre, les plantes, les termites, réduisant ainsi le coût du travail pour maintenir et construire les champs de buttes. C'est une forme d'agriculture qui maintient la biodiversité des savanes. C'est aussi un rare exemple d'agriculteurs qui travaillent avec les paysages humides et pas à leur encontre.

\section{En Afrique, où l'agriculture sur champs surélevés vit encore}

Mais il était frustrant de travailler avec des vestiges d'agriculture de champs surélevés. Il fallait donc quitter l'Amérique du Sud, où ce système est quasiment disparu depuis la conquête européenne, et aller en Afrique, où ce type d'agriculture est encore pratiqué dans plusieurs endroits aujourd'hui.

Par exemple, dans la cuvette du Congo, un paysage hybride qui est l'œuvre d'ingénieurs humains et non humains (figure 10). Le paysage semble avoir commencé avec des grandes termitières qui ont été utilisées comme noyaux dans un réseau d'autres structures. Par exemple, il y a des billons linéaires ou curvilinéaires (pistes? champs surélevés? barrages à poissons? on ne sait pas), entre les termitières. Il y a aussi beaucoup d'étangs qui proviennent des borrow pits, quand les gens ont déplacé la terre pour faire les billons. On ne connaît pas la fonction de ces étangs. Ont-ils servis pour stocker du poisson vivant durant la saison sèche? 


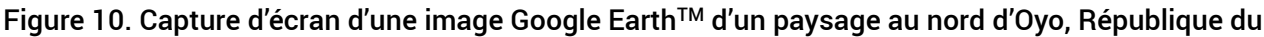
Congo, montrant des structures rondes d'origine probablement naturelle (grandes termitières) intégrées dans un réseau de structures d'origine anthropique

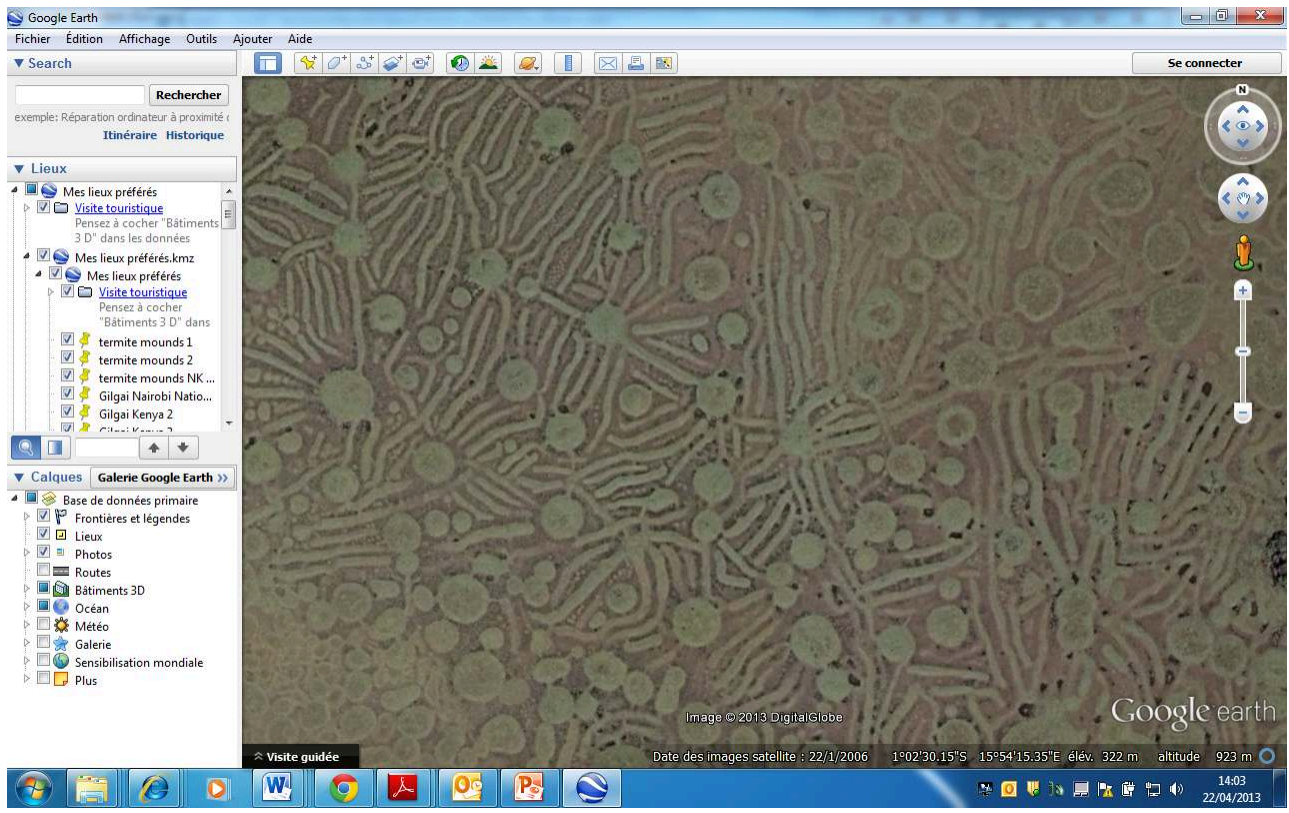

Si on va suffisamment loin de la ville d'Oyo, moteur d'un exode rural dans cette région, on voit que cette agriculture est encore pratiquée aujourd'hui, avec des cultures plantées sur de grosses buttes dans les savanes inondables (figure 11).

Figure 11. Champs surélevés actuellement cultivés (ici plantés avec du manioc), près de Mossaka, République du Congo.

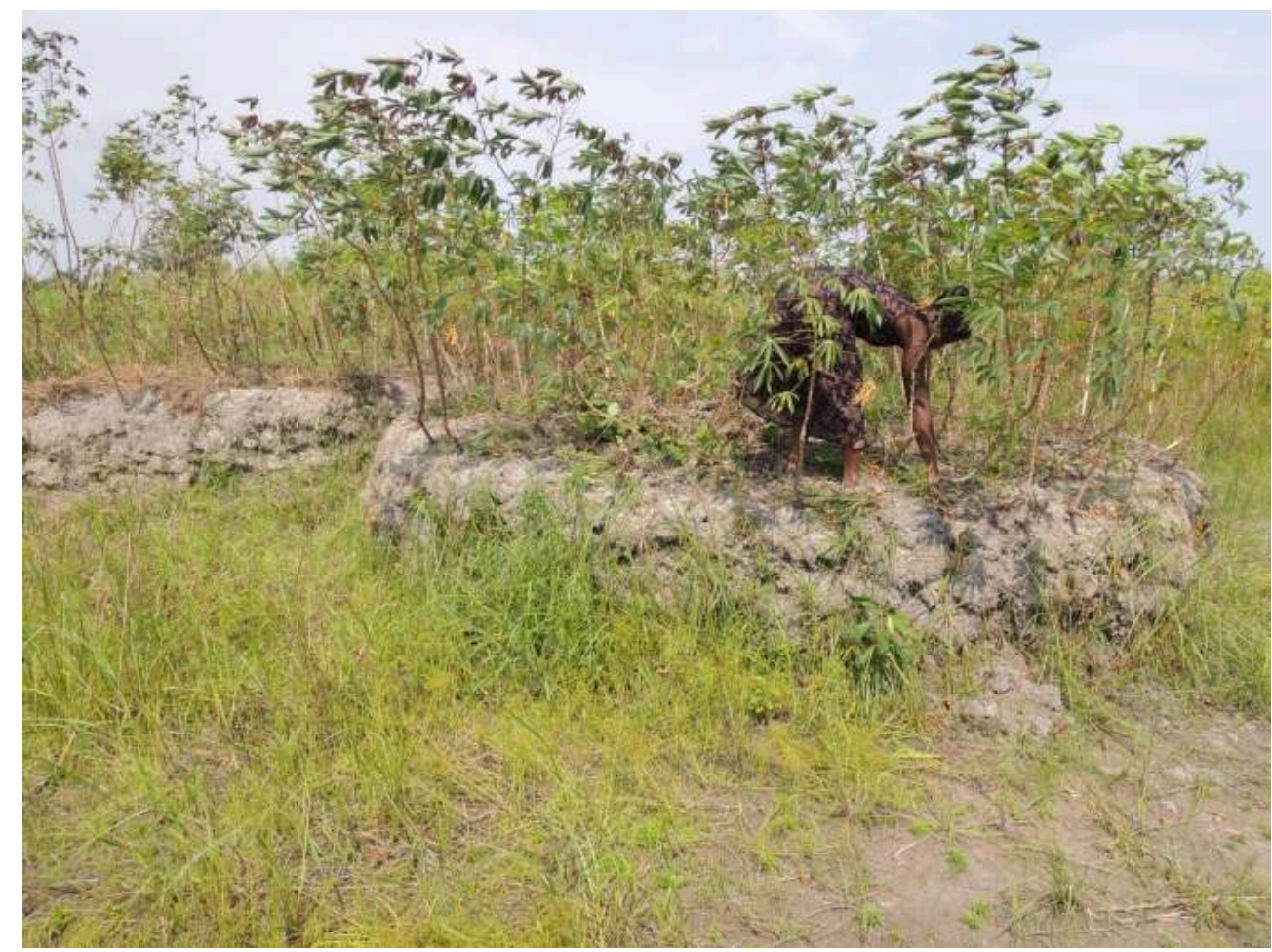

Photographie : Doyle McKey 
Nous avons fait deux petites missions en Afrique, une au Congo et une en Zambie, pour étudier ces systèmes vivants d'agriculture sur champs surélevés, et pour explorer les interactions entre les agriculteurs et les structures surélevées d'origine naturelle dans ces sites. Voici quelques diapositives du deuxième site, sur lequel nous nous sommes rendus pour la première fois en septembre 2013: dans le bassin de Bangweulu en Zambie. On voit des paysages à buttes naturelles, des petites et des grosses termitières (figure 12, photo de gauche). Comme au Congo, ce sont des paysages hybrides parce que les grosses termitières sont souvent modifiées en champs surélevés (figure 12, photo de droite).

Figure 12. Paysages à buttes dans le Bassin de Bangweulu, Zambie.
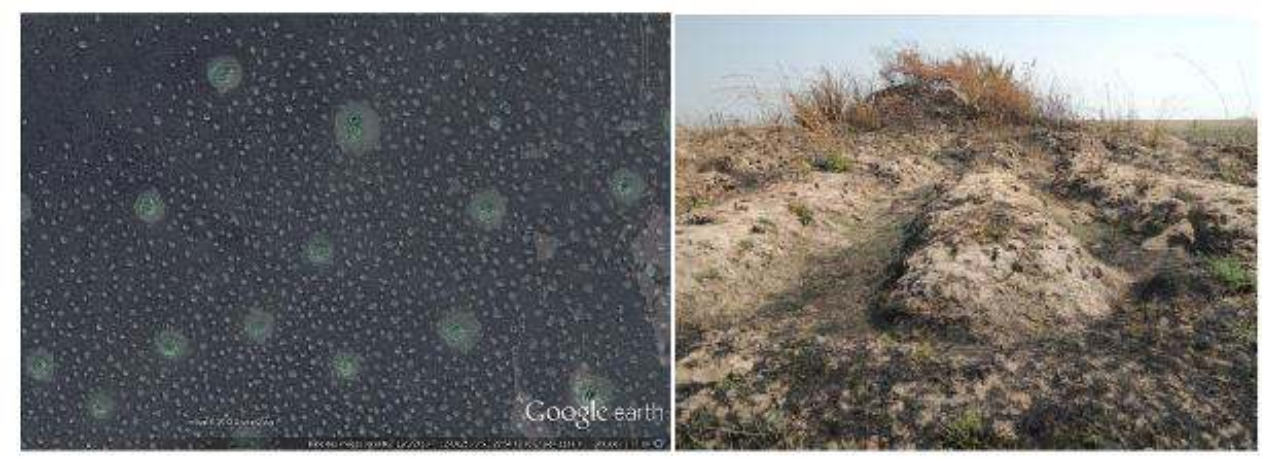

À gauche, une capture d'écran de Google Earth ${ }^{\mathrm{TM}}$ montrant un paysage marqué par des termitières de deux espèces de termite, une faisant de grandes buttes, l'autre des petites buttes. Les grandes termitières sont souvent aménagées en champs surélevés (photo à droite, photographie : Doyle McKey)

Dans ces paysages hybrides, on voit des petites termitières d'une espèce, des grandes termitières d'une autre, sur lesquelles sont souvent construits des champs surélevés. Dans d'autres parties du Bassin de Bangweulu, de grands champs surélevés sont construits sans l'aide des termites (figure 13). 
Figure 13. Dans le Bassin de Bangweulu se trouvent aussi de grands champs surélevés d'origine purement anthropique, sans l'intervention des termites.

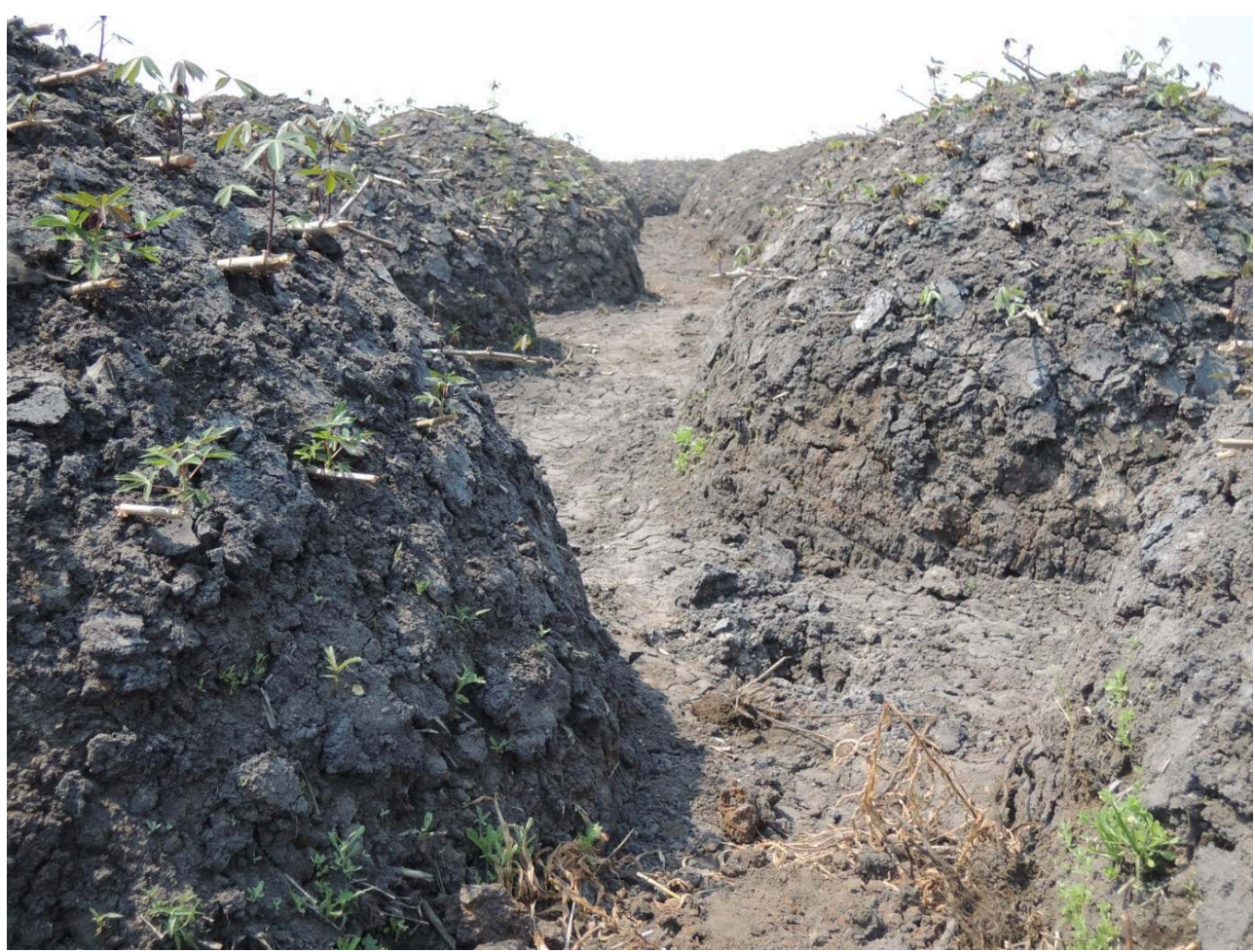

Ceux-ci sont hauts d'environ 1,70 m. Le manioc est planté à partir de $70 \mathrm{~cm}$, pour échapper aux inondations durant la saison des hautes eaux.

Photographie : Doyle McKey

32 Il y a beaucoup de choses à faire en étudiant ces systèmes, nous en sommes juste au début. Pour la première fois, on est dans un paysage où il y a des agriculteurs à qui on peut demander ce qui se passe. Il est clair que, pour l'instant, nous n'avons que des données sur l'aspect biophysique. Pour ce qui intéresse plus particulièrement l'anthropologie, par exemple la manière dont les humains perçoivent ces environnements, nous en sommes encore plutôt à nous poser des questions. Est-ce qu'il y a un biomimétisme à l'échelle de l'écosystème? Quelles sont les perceptions des agriculteurs de ces paysages d'origine naturelle ? Est-ce qu'il y a une convergence entre leur pensée et celles des écologues? Ont-ils des explications pour les processus qui sous-tendent l'origine des structures naturelles? Quand ils reconstruisent leur propre paysage culturel, est-ce que les agriculteurs imitent de façon consciente (ou pas) ces paysages d'origine naturelle? Si oui, est-ce qu'ils imitent les structures, pour essayer d'imiter par hasard les processus, ou est-ce qu'ils essaient d'imiter des processus pour lesquels ils ont des explications? Ou est-ce que c'est quelque chose de différent, des adaptations convergentes complètement indépendantes de différents organismes? Les termites n'ont pas dû observer les vers de terre pour apprendre à construire leurs buttes, et vice versa: les deux ont construit leurs buttes de façon indépendante. Pourquoi les humains ne seraient-ils pas capables eux aussi d'une invention indépendante des buttes, sans passer par l'observation des termites ou des vers de terre?

33 Le jeu de mot ne passe pas en anglais. Mais on peut se demander si c'est du vers (des vers [de terre]) ou de la prose? Le Bourgeois Gentilhomme de Molière dit : « My god, I've 
been speaking prose for forty years and I never knew it ». Exactement comme le Bourgeois Gentilhomme, peut-être les agriculteurs ont-ils eu une démarche biomimétique pendant des siècles sans en être conscients: un patron similaire ne suppose pas nécessairement un projet d'imitation.

Ce sont des paysages hybrides. On a montré des exemples où les ingénieurs du sol bâtissent et maintiennent les paysages créés par les humains. C'est vrai aussi dans le sens inverse. Les humains intègrent des structures naturelles dans les paysages hybrides. Une autre question se pose : est-ce que l'organisation de ces paysages reflète un projet de la société ou est-ce que les paysages d'origine humaine, comme les paysages d'origine naturelle, résultent de processus auto-organisés, dont l'origine n'est peut-être pas expliquée par les dynamiques écologiques, mais par des dynamiques sociales? Par exemple, on peut penser au modèle de Thomas Schelling (voir surtout Schelling, T. C. 1971 "Dynamic models of segregation", Journal of Mathematical Sociology, 1(2) : 143-186) pour expliquer l'auto-organisation des patrons de ségrégation raciale dans les paysages urbains.

\section{ABSTRACTS}

In a wide range of environments - but which all have in common the scarcity of some key resource that is crucial for life- there have emerged ecosystems that show remarkably regular spatial patterns in the distribution of vegetation, in topography, or both. These patterns have been shown to result from the activities of organisms that can be classed as "ecosystem engineers." In these constraining environments, "engineer" organisms, acting in concert with physical mechanisms, convert an initially homogeneous landscape into a landscape composed of two kinds of patches, one kind in which the crucial resource is concentrated, the other kind in which the crucial resource is depleted. Thus, in environments where a crucial resource is generally scarce, engineer organisms create patches of habitat in which the resource is relatively abundant. This allows organisms to persist in environments that would otherwise be marginal (or worse) for their survival. The process by which this occurs is termed "spatial selforganization" and the phenomenon is called "self-organized patchiness." In some of the environments where strikingly patterned, self-organized ecosystems occur, farmers construct cultural landscapes that are remarkably similar to the constructions of the "engineer" organisms. This resemblance between artefacts made by humans and constructions made by non-human living beings raises numerous questions. Does the resemblance reflect independent convergence on similar adaptive solutions to constraining environments, or do human and nonhuman engineers interact in one or more ways? The objective of this presentation is to explore these questions. 


\section{AUTHOR}

\section{DOYLE MCKEY}

Doyle McKey is professor of ecology at the Université Montpellier 2 and is a senior member of the Institut Universitaire de France. He studies interactions between plants and animals, and between plants and humans, in tropical ecosystems. His widely cited work covers diverse topics including the interactions between plants and vertebrate frugivore seed-dispersers, the theory of plant defence against herbivores, the ecology of protective ant-plant mutualisms, plant evolution under domestication, chemical ecology of plant-human interactions, historical ecology and landscape domestication. The transdisciplinary approach he pursues has required forging collaborations with researchers in a great diversity of fields in natural and social sciences. He has published in over 70 different journals, including Science, PNAS, and Current Biology, as well as top journals in ecology, evolution, botany, agronomy, soil science, archaeology and anthropology. Research he led on the domestication of manioc by Amerindian farmers in South America has transformed our ideas about the evolution of clonally propagated crops. For the past six years, he has directed an interdisciplinary project on the ecology of seasonally flooded savannas. He first examined the ecological legacy of pre-Columbian raised-field agriculture in South American seasonal wetlands, showing how this ancient agricultural activity continues to condition the present-day functioning of these ecosystems. A second component of the project focuses on the ecology of mound-field landscapes of natural origin in seasonal wetlands, examining mechanisms leading to spatial regularity and identifying analogies, and possible interactions, between the functioning of these ecosystems and that of agricultural raised fields. A third component examines present-day raised-field farming systems in Africa. This work has demonstrated synergies between human engineering and the actions of natural "soil engineers", and has shown that raised-field agriculture is but one component of a multi-activity system, and cannot be understood in isolation from other components of the system, notably floodplain fishing. [doyle.mckey [@] cefe.cnrs.fr] 